\title{
MAXIMUM EIGENVALUE DETECTION FOR SPECTRUM SENSING UNDER CORRELATED NOISE
}

\author{
Shree Krishna Sharma, Symeon Chatzinotas, and Björn Ottersten \\ SnT - securityandtrust.lu, University of Luxembourg \\ Email:\{shree.sharma, symeon.chatzinotas, bjorn.ottersten\}@uni.lu
}

\begin{abstract}
Herein, we consider the problem of detecting primary users' signals in the presence of noise correlation, which may arise due to imperfections in filtering and oversampling operations in a Cognitive Radio (CR) receiver. In this context, we study a Maximum Eigenvalue (ME) detection technique using recent results from Random Matrix Theory (RMT) for characterizing the distribution of the maximum eigenvalue of a class of sample covariance matrices. Subsequently, we derive a theoretical expression for a sensing threshold as a function of the probability of false alarm and evaluate the sensing performance in terms of probability of correct decision. It is shown that the proposed approach significantly improves the sensing performance of the ME detector in correlated noise scenarios.
\end{abstract}

Index Terms - Spectrum Sensing, Noise Correlation, Cognitive Radio, Random Matrix theory

\section{INTRODUCTION}

Cognitive Radio (CR) communications is considered a promising solution in order to address the spectrum scarcity problem caused by the remarkable growth of wireless broadband devices and services competing for the limited usable spectrum $[1,2]$. One of the key functions of a CR is to adjust its system parameters based on the spectral usage knowledge of its surrounding environment so that the normal operation of licensed primary systems is not affected. For this purpose, Spectrum Sensing (SS) is an important mechanism required by a CR in order to detect spectral holes in different dimensions such as frequency, space, area, polarization and angular domains $[3,4,5]$. In this context, several SS techniques such as Energy Detection (ED), matched filter based detection, cyclostationary feature based detection, covariance based detection, eigenvalue based detection have been proposed in the literature for sensing the presence of a Primary User (PU) [3]. Furthermore, in order to enhance the SS efficiency in wireless fading channels, a multidimensional $\mathrm{CR}$ receiver has been studied considering multiple receive dimensions at the CR receiver in the form of multiple antennas, oversampled branches and cooperative nodes $[6,7,8,10]$. These methods are mostly based on the statistics of the eigenvalues of the received signal's covariance matrix and use recent results from Random Matrix Theory (RMT). The main advantage of the eigenvalue based approach in practical scenarios is that it does not require any prior information about the PU's signal and the channel.

1) Related Work: RMT has received considerable attention in the SS literature for studying different eigenvalue based algorithms $[11,7]$. The contributions related to the eigenvalue based sensing exploiting RMT methods include [6, 7, 8, 10, 11, 12, 13, 14, 15]. The existing techniques can be categorized into Maximum Eigenvalue (ME) based [14], Signal Condition Number (SCN) based [6,
$7,10,12]$ and Scaled Largest Eigenvalue (SLE) based [15, 16]. The ME based detection has been proposed in [14], which utilizes the maximum eigenvalue of the received signal's covariance matrix in order to detect the presence of the PU signal. The authors in [11] use the Marcenko-Pastur (MP) law to test a binary hypothesis assuming the presence of the white noise. In [6], semi-asymptotic Maximum to Minimum Eigenvalue (MME) and Energy with Minimum Eigenvalue (EME) algorithms for SS have been proposed using the combination of the MP and Tracy-Widom (TW) distributions and a ratio based technique has been proposed in [12] using the TracyWidom Curtiss (TWC) distribution. The contribution in [17] provides a general framework for the Cumulative Distribution Function (CDF) of the SCN of different classes of Wishart matrices. Furthermore, in [18], analytic expressions for the Probability Density Function (PDF) and CDF of the ratio of the maximum eigenvalue to the trace of complex Wishart matrices with arbitrary dimensions have been derived. Moreover, the recent contribution in [9] studies an information-plus-noise transmission model to perform statistical eigen-inference under the unknown noise correlation pattern.

In our previous work [19], the asymptotic analysis of different eigenvalue-based blind sensing techniques such as SLE, SCN, John's detection and Spherical Test (ST) based detection has been carried out and it has been shown that noise correlation significantly degrades the sensing performance of these techniques. Considering the presence of noise correlation, the contribution in [10] proposes a new sensing threshold for enhancing sensing performance based on a theoretical analysis under a noise only hypothesis. Subsequently, a Signal to Noise Ratio (SNR) estimation technique has been proposed in order to estimate the PU SNR in the presence of correlated noise based on the analysis under signal plus noise hypothesis. Furthermore, the contribution in [21] studies an SNR estimation technique for a multidimensional CR receiver under correlated channel/noise based on the maximum eigenvalue of the received signal's sample covariance matrix.

2) Contributions: Most of the eigenvalue based techniques in the literature assume the presence of the white noise at the CR receiver. However, in practice, the noise may be correlated due to imperfections in filtering interference or oversampling operations $[6,21]$. Since the value of decision statistics in the presence of noise correlation deviates from its value in uncorrelated scenarios, a sensing threshold proposed for the uncorrelated scenario may not be suitable for sensing in the presence of noise correlation [10]. While applying the sensing threshold designed for the uncorrelated scenario in correlated scenarios, the value of the probability of a false alarm $\left(P_{f}\right)$ deviates from the target $P_{f}$ value used for calculating the sensing threshold, resulting in degraded sensing performance. To address this issue, we consider the problem of detecting the PU's signal in the presence of noise correlation at the CR receiver. We analyze the considered correlated scenario using a tilted semicircular distri- 
bution for modeling the noise covariance matrix as in [22], which resembles the exponential covariance model considered in various previous works [10, 13, 24, 23]. Furthermore, we use recent RMT results on the universality of the maximum eigenvalue of a class of sample covariance matrices from [27] in order to derive the expression for the sensing threshold, which is the main contribution of this paper. Subsequently, the proposed threshold is used for sensing the presence of a PU signal in practical scenarios where noise correlation is always present. Moreover, the performance of the proposed approach is evaluated in terms of the false alarm deviation and probability of correct decision. We define the false alarm deviation as the absolute difference between the observed $P_{f}$ and the target $P_{f}$ used for determining the sensing threshold. Furthermore, we define the probability of correct decision as the number of correct decisions out of the total considered cases under both hypotheses as in $[10,13]$.

The remainder of this paper is structured as follows: Section 2 provides the signal and channel model. Section 3 presents the analysis for the correlated noise case considering a tilted semicircular distribution and derives the expressions for the mean and variance. Section 4 proposes a sensing threshold for the ME detection in the presence of noise correlation. Section 5 studies the sensing performance of the proposed approach with the help of numerical results. Section 6 concludes the paper.

3) Notation Throughout this paper, boldface upper and lower case letters are used to denote matrices and vectors respectively, $\mathbb{E}[\cdot]$ denotes the expectation, $(\cdot)^{T}$ denotes the transpose matrix, $(\cdot)^{H}$ denotes the conjugate transpose matrix, $(\cdot)^{*}$ represents the complex conjugate, $\mathbb{C}$ denotes complex numbers, $|\cdot|$ denotes the absolute operation, $\mathbf{R}_{\mathbf{X}}$ represents the covariance matrix of $\mathbf{X}$, and $\lambda_{\max }(\cdot)$ denotes the maximum eigenvalue of a matrix.

\section{SIGNAL MODEL}

Let us consider a single cognitive user and a single PU for simplicity of analysis. Let $N$ be the number of samples analyzed by the cognitive user for the decision process and $\tau$ the sensing duration. Let $M$ be the number of receive dimensions in a CR receiver. From a system-model point of view, this factor can be considered as the number of antennas in a multiantenna-based CR receiver and/or the number of oversampled branches in an oversampling-based sensing model as considered in $[6,7,10]$.

We assume that the transmitted symbols are independent and identically distributed (i.i.d.) complex circularly symmetric (c.c.s.) symbols, the noise samples in each receive dimension are independent and c.c.s. Gaussian but are correlated across receive dimensions. Depending on the nature of wireless channels and the relation between sensing duration $\tau$ and symbol duration $T_{s}$, different signal models can be considered [13]. In this work, we assume that $\tau>T_{s}$ and the channel remains constant during the period of sensing. The $M \times N$ received signal model in this case can be written as:

$$
\mathbf{Y}=\mathbf{h s}+\hat{\mathbf{Z}},
$$

where $\mathbf{h}=\left[h_{1}, h_{2}, \ldots, h_{M}\right]^{T}$ is an $M \times 1$ vector with $h_{m}$ representing a channel coefficient between the $m$ th receive branch and the $\mathrm{PU}, \mathbf{s}$ is an $1 \times N$ PU transmitted signal i.e., $\mathbf{s}=\left[s_{1}, s_{2}, \ldots, s_{N}\right]$, with $s_{i}$ being a transmitted symbol with power $p_{i}=\mathbb{E}\left[s_{i}^{2}\right]$, and $\hat{\mathbf{Z}} \sim \mathcal{C N}\left(0, \mathbf{R}_{\hat{\mathbf{z}}}(N)\right)$ is the colored noise. Let us denote the hypotheses of the presence and absence of the PU signal by $\mathbb{H}_{1}$ and $\mathbb{H}_{0}$ respectively. The binary hypothesis testing problem for deciding the presence of a PU signal can be written as:

$$
\mathbb{H}_{0}: \mathbf{Y}=\hat{\mathbf{Z}}, \mathbb{H}_{1}: \mathbf{Y}=\mathbf{h s}+\hat{\mathbf{Z}}
$$

Let us define the sample covariance matrices of the received signal and the noise as: $\mathbf{R}_{\mathbf{Y}}(N)=\frac{1}{N} \mathbf{Y} \mathbf{Y}^{H}$ and $\mathbf{R}_{\hat{\mathbf{Z}}}(N)=\frac{1}{N} \hat{\mathbf{Z}} \hat{\mathbf{Z}}^{H}$. Under the $\mathbb{H}_{0}$ hypothesis, the sample covariance matrix of the received signal becomes equal to the sample covariance matrix of the noise i.e., $\mathbf{R}_{\mathbf{Y}}(N)=\mathbf{R}_{\hat{\mathbf{Z}}}(N)$. Since our detection method depends only on statistics of $\mathbf{R}_{\hat{\mathbf{Z}}}(N)$, all the signal types can be considered under this framework.

1) Noise Correlation Modeling: The causes of noise correlation for a multi-dimensional CR in practical scenarios have been discussed in [21]. In this study, we consider noise correlation across the receive dimensions and not across the temporal dimension as mentioned before. To model this scenario, we consider the one-sided noise correlation model as in [10]. The correlated noise $\hat{\mathbf{Z}}$ is modeled as: $\hat{\mathbf{Z}}=\boldsymbol{\Theta}^{1 / 2} \mathbf{Z}$, where $\mathbf{Z}$ is an $M \times N$ matrix with i.i.d. complex Gaussian entries having zero mean and variance $\sigma_{z}^{2}$, representing the white noise and $\boldsymbol{\Theta}^{1 / 2} \boldsymbol{\Theta}^{1 / 2}=\boldsymbol{\Theta}=\mathbb{E}\left[\hat{\mathbf{Z}} \hat{\mathbf{Z}}^{H}\right]$. It can be noted that since $\mathbf{Z} \sim \mathcal{C N}\left(0, \sigma_{z}^{2} \mathbf{I}\right), \mathbf{Z Z}^{H}$ follows an uncorrelated Wishart distribution i.e., $\mathbf{Z Z} \mathbf{Z}^{H} \sim \mathcal{W}_{M}\left(\mathbf{R}_{\mathbf{Z}}, N\right)$. We consider the normalization $(1 / M) \operatorname{trace}\{\boldsymbol{\Theta}\}=1$ in order to ensure that $\boldsymbol{\Theta}$ does not affect the noise power. The components of $\Theta$ using an exponential covariance model can be defined as [24]:

$$
\theta_{i j} \sim \begin{cases}\rho^{(j-i)}, & i \leq j \\ \left(\rho^{(i-j)}\right)^{*}, & i>j\end{cases}
$$

where $\theta_{i j}$ is the $(i, j)$ th element of $\boldsymbol{\Theta}$ and $\rho \in \mathbb{C}$ is the correlation coefficient with $|\rho| \leq 1$.

2) ME based Detection: The decision statistics for this detection method is the ratio of the maximum eigenvalue to the noise variance, also called Roy's Largest Root Test (RLRT) in the literature [25]. The performance analysis of this detector has been carried out in $[25,26]$ under white noise scenarios and it has been shown that this detector outperforms the ED technique for Gaussian signals. Let $\gamma_{c}$ be the sensing threshold used for the decision process in the considered correlated scenario. The binary hypothesis based on the ME method can be expressed as:

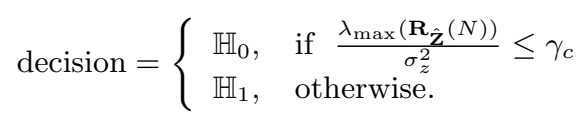

\section{ANALYSIS UNDER $\mathbb{H}_{0}$ HYPOTHESIS}

Let $\hat{\mathbf{R}}_{\mathbf{Z}}(N)=\frac{N}{\sigma_{z}^{2}} \mathbf{R}_{\mathbf{Z}}(N)$ denote the normalized sample covariance matrix under the white noise scenario. Under the assumption $N, M \rightarrow \infty$ with $N / M \rightarrow \beta$ and under white noise (complex) scenarios, the random variable $\frac{\lambda_{\max }\left(\hat{\mathbf{R}}_{\mathbf{Z}}(N)\right)-\nu}{\sigma}$ with $\sigma=(\sqrt{M}+$ $\sqrt{N})(1 / \sqrt{M}+1 / \sqrt{N})^{\frac{1}{3}}$ and $\nu=(\sqrt{M}+\sqrt{N})^{2}$ converges to the Tracy-Widom distribution of order $2\left(\mathrm{TW}_{2}\right)$ [6, 12, 29], which can be stated as:

$$
\frac{\lambda_{\max }\left(\hat{\mathbf{R}}_{\mathbf{Z}}(N)\right)-\nu}{\sigma} \Longrightarrow \mathrm{TW}_{2} .
$$

The $\mathrm{TW}_{2}$ distribution can be defined as [30]:

$$
F_{2}=\exp \left(-\int_{s}^{+\infty}(x-s) q^{2}(x) d x\right),
$$

where $q(s)$ is the solution of the Painlevé II differential equation $q^{\prime \prime}(s)=s q(s)+2 q^{3}(s)$ satisfying the condition $q(s) \sim-A i(s)$ (the Airy function) for $s \rightarrow+\infty$. Due to involvement of a nonlinear differential equation, it is generally complex to evaluate the TW distribution. However, due to its important application in RMT, this 
distribution has been widely studied and tables for the functions are available in the literature [29].

The contributions in $[6,14]$ have exploited the above result in order to derive the expression for sensing threshold in terms of $P_{f}$. The eigenvalue-based detection technique has been analyzed in the presence of noise correlation in [10] using the SCN based asymptotic approach. However, the asymptotic threshold used in [10] does not depend on the value of $P_{f}$. In practical scenarios, we may need to vary the decision threshold based on the acceptable value of $P_{f}$. In this context, we are interested in finding out a theoretical expression for the sensing threshold as a function of $P_{f}$ in the presence of noise correlation for the case of the ME detector using recent RMT results obtained in [27].

Under the $\mathbb{H}_{0}$ hypothesis, the sample covariance matrix $\mathbf{R}_{\mathbf{Y}}(N)$ in the presence of noise correlation can be written as:

$$
\mathbf{R}_{\mathbf{Y}}(N)=\mathbf{R}_{\hat{\mathbf{Z}}}(N)=\frac{1}{N} \Theta^{1 / 2} \mathbf{Z} \mathbf{Z}^{H} \Theta^{1 / 2} .
$$

To model the distribution of the noise covariance matrix $\Theta$, we use tilted semicircular distribution as in $[10,22]$. The tilted semicircular distribution can be written as [22]:

$$
f_{\Theta}(\lambda)=\frac{1}{2 \pi \mu \lambda^{2}} \sqrt{\left(\frac{\lambda}{\sigma_{1}}-1\right)\left(1-\frac{\lambda}{\sigma_{2}}\right)},
$$

with $\sigma_{1} \leq \lambda \leq \sigma_{2}$ and $\mu=\frac{\left(\sqrt{\sigma_{2}}-\sqrt{\sigma_{1}}\right)^{2}}{4 \sigma_{1} \sigma_{2}}$.

Let us define the normalized sample covariance matrix under the correlated noise scenario as: $\hat{\mathbf{R}}_{\hat{\mathbf{Z}}}(N)=\frac{1}{\sigma_{z}^{2}} \mathbf{R}_{\hat{\mathbf{Z}}}(N)$. From [Theorem 1.5, [27]], the normalized sample covariance matrix $\hat{\mathbf{R}}_{\hat{\mathbf{Z}}}(N)^{1}$ follows the $\mathrm{TW}_{2}$ distribution in the following way

$$
N^{2 / 3}\left(\frac{\lambda_{\max }\left(\hat{\mathbf{R}}_{\hat{\mathbf{Z}}}(N)\right)-\nu_{c}}{\sigma_{c}}\right) \Longrightarrow T W_{2},
$$

where $\sigma_{c}^{3}=\frac{1}{c^{3}}\left(1+\frac{1}{\beta} \int\left(\frac{\lambda c}{1-\lambda c}\right)^{3} d F_{\Theta}(\lambda)\right)$, and $\nu_{c}=\frac{1}{c}\left(1+\frac{1}{\beta} \int \frac{\lambda c}{1-\lambda c} d F_{\Theta}(\lambda)\right)$. The value of $c$ in the above expressions can be obtained by solving the following equation $[27,28]$

$$
\int\left(\frac{\lambda c}{1-\lambda c}\right)^{2} d F_{\Theta}(\lambda)=\int\left(\frac{\lambda c}{1-\lambda c}\right)^{2} f_{\Theta}(\lambda) d \lambda=\beta .
$$

It should be noted that the value of $c$ is unique in the range $c \in$ $\left[0,1 / \lambda_{\max }(\boldsymbol{\Theta})\right]$. Substituting the distribution of $\boldsymbol{\Theta}$ from (8) in (10) yields the following expression

$$
\int\left(\frac{\lambda c}{1-\lambda c}\right)^{2} \frac{1}{2 \pi \mu \lambda^{2}} \sqrt{\left(\frac{\lambda}{\sigma_{1}}-1\right)\left(1-\frac{\lambda}{\sigma_{2}}\right)}-\beta=0 .
$$

Furthermore, the ratio $\sigma_{2} / \sigma_{1}$ in (8) can be written as [22]: $\frac{\sigma_{2}}{\sigma_{1}}=\frac{(1+\rho)^{2}}{(1-\rho)^{2}}$. Using the above relation and $\sigma_{1} \sigma_{2}=1$, the values of $\sigma_{1}$ and $\sigma_{2}$ can be written as: $\sigma_{1}=\frac{1-\rho}{1+\rho}, \quad \sigma_{2}=\frac{1+\rho}{1-\rho}$. Substituting the values of $\sigma_{1}$ and $\sigma_{2}$ in (11) and taking integration limits from $\sigma_{1}$ to $\sigma_{2}$, (11) can be written as:

$$
\frac{c^{2}}{2 \pi \mu} \int_{\sigma_{1}}^{\sigma_{2}} \frac{1}{(1-\lambda c)^{2}} \sqrt{-\lambda^{2}-1+2 \lambda \mu_{1}} d \lambda-\beta=0,
$$

${ }^{1}$ This normalization is used by considering the fact that the sample covariance matrix of the form (7) with variance $1 / N$ follows Theorem 1.5 in [27]. where $\mu_{1}=\left(1+\rho^{2}\right) /\left(1-\rho^{2}\right)$. Similarly, the expressions for $\nu_{c}$ and $\sigma_{c}^{3}$ can be written as:

$$
\begin{aligned}
\nu_{c} & =\frac{1}{c}\left(1+\frac{c}{2 \pi \mu \beta} \int_{\sigma_{1}}^{\sigma_{2}} \frac{1}{(1-\lambda c) \lambda} \sqrt{-\lambda^{2}-1+2 \lambda \mu_{1}} d \lambda\right), \\
\sigma_{c}^{3} & =\frac{1}{c^{3}}\left(1+\frac{c^{3}}{2 \pi \mu \beta} \int_{\sigma_{1}}^{\sigma_{2}} \frac{\lambda}{(1-\lambda c)^{3}} \sqrt{-\lambda^{2}-1+2 \lambda \mu_{1}} d \lambda\right) .
\end{aligned}
$$

\section{DERIVATION OF SENSING THRESHOLD}

In this section, we derive an analytical expression for the sensing threshold for the considered ME detection following the approach used in $[6,14]$. In practice, a CR receiver should be able to detect the presence of PU signals with higher probability of detection $\left(P_{d}\right)$ and low $P_{f}$. However, there exists a trade-off between these two parameters and the sensing threshold should be selected to meet the required value of $P_{f}$. Since it's not practical to set a threshold based on the value of $P_{d}$ due to absence of the knowledge about the PU signal, the threshold is usually determined based on the predefined value of $P_{f}$. The contribution in [14] proposes a sensing threshold for the ME detection for the white noise case, which can be written as $[12,14]$ :

$$
\gamma=\frac{(\sqrt{N}+\sqrt{M})^{2}}{N}\left(1+\frac{(\sqrt{N}+\sqrt{M})^{-2 / 3}}{(N M)^{1 / 6}} F_{2}^{-1}\left(1-P_{f}\right)\right) .
$$

In correlated noise scenarios, the detection with the threshold in (15) may not provide perfect sensing performance. For this purpose, we propose a new threshold for the ME detector using the analysis presented in Section 3. The probability of false alarm of the ME based detection is given by

$$
\begin{array}{r}
P_{f}=P\left(\lambda_{\max }\left(\mathbf{R}_{\hat{\mathbf{Z}}}\right)>\gamma_{c} \sigma_{z}^{2}\right)=P\left(\sigma_{z}^{2} \lambda_{\max }\left(\hat{\mathbf{R}}_{\hat{\mathbf{Z}}}\right)>\gamma_{c} \sigma_{z}^{2}\right), \\
=P\left(N^{2 / 3} \frac{\lambda_{\max }\left(\hat{\mathbf{R}}_{\hat{\mathbf{Z}}}\right)-\nu_{c}}{\sigma_{c}}>N^{2 / 3} \frac{\gamma_{c}-\nu_{c}}{\sigma_{c}}\right) .
\end{array}
$$

Subsequently, using the condition (9), (16) can be written as:

$$
P_{f}=1-F_{2}\left(N^{2 / 3}\left(\frac{\gamma_{c}-\nu_{c}}{\sigma_{c}}\right)\right) .
$$

Equivalently, the above expression can be written as:

$$
N^{2 / 3}\left(\frac{\gamma_{c}-\nu_{c}}{\sigma_{c}}\right)=F_{2}^{-1}\left(1-P_{f}\right)
$$

Subsequently, the value of $\gamma_{c}$ can be calculated as:

$$
\gamma_{c}=\frac{\sigma_{c} F_{2}^{-1}\left(1-P_{f}\right)+N^{2 / 3} \nu_{c}}{N^{2 / 3}} .
$$

\section{NUMERICAL RESULTS}

We analyze the performance of the proposed sensing approach in terms of false alarm deviation and the probability of correct decision. The false alarm deviation is defined as: $\left|P_{f t}-P_{f s}\right|$, where $P_{f t}$ is the target value of $P_{f}$, which is usually chosen based on the sensing requirements of a wireless standard and $P_{f s}$ is the simulated value of $P_{f}$ observed during the decision process. The probability of correct decision refers to the ratio of the number of correct decisions to the total number of considered cases under both hypotheses and is 


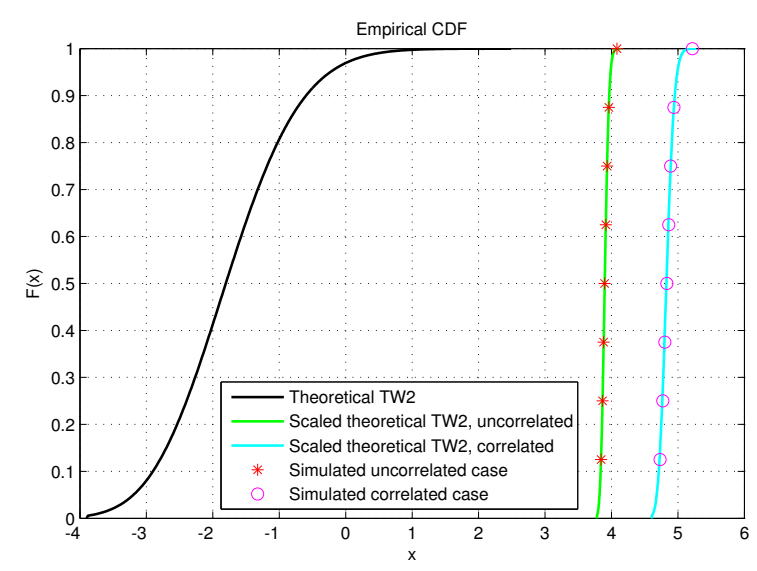

Fig. 1. Theoretical and simulated CDF plots for different random variables $(N=300, \beta=1, \rho=0.5)$

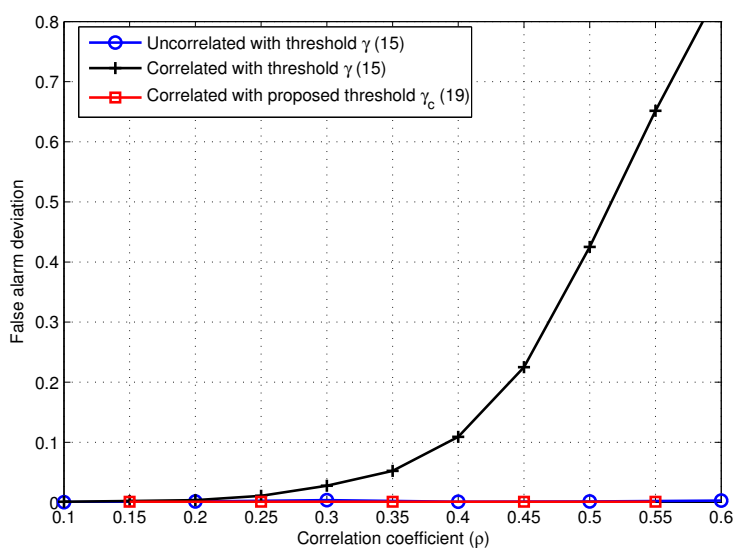

Fig. 2. False alarm deviation versus $\rho\left(N=100, \beta=2, P_{f}=0.001\right)$

defined as [10, 19]: $\left(P_{d}+\left(1-P_{f}\right)\right) / 2$, where $P_{d}$ and $1-P_{f}$ denote the number of correct decisions out of the total considered cases under $\mathbb{H}_{1}$ and $\mathbb{H}_{0}$ hypotheses respectively. The number of cases considered in the presented simulation results were $10^{3}$. We consider a flat fading Rayleigh fading channel in our simulation model and its coefficients are generated from random complex numbers whose real and imaginary components are i.i.d. Gaussian variables. We assume perfect knowledge of the noise variance as in [14, 25, 26]. In practice, this knowledge can be acquired by using noise covariance estimation techniques as in [32].

In order to validate the theoretical analysis presented in Section 3, we present CDF plots for the following cases in Fig. 1: (i) Theoretical $\mathrm{TW}_{2}$ distribution from (6), (ii) Scaled theoretical $\mathrm{TW}_{2}$ for the uncorrelated case, obtained by scaling the $\mathrm{TW}_{2}$ distribution based on (5) (iii) Scaled theoretical $\mathrm{TW}_{2}$ for the correlated case, obtained by scaling the $\mathrm{TW}_{2}$ distribution based on (9), (iv) Simulated uncorrelated case, obtained by considering $10^{3}$ realizations of $\lambda_{\max }\left(\mathbf{R}_{\mathbf{Z}}(N)\right)$, and (v) Simulated correlated case, obtained by taking $10^{3}$ realizations of $\lambda_{\max }\left(\mathbf{R}_{\hat{\mathbf{Z}}}(N)\right)$. From the figure, we note that the CDF of $\lambda_{\max }\left(\mathbf{R}_{\mathbf{Z}}(N)\right)$ matches perfectly with the theoretical $\mathrm{TW}_{2}$ distribution, scaled based on (5) and the CDF of $\lambda_{\max }\left(\mathbf{R}_{\hat{\mathbf{z}}}(N)\right)$ matches perfectly with the theoretical $\mathrm{TW}_{2}$ distribution, scaled based on (9). From this result, it can be concluded that the $\mathrm{TW}_{2}$ distribution can be used for deriving the sensing threshold under the considered correlated scenario considering the mean and

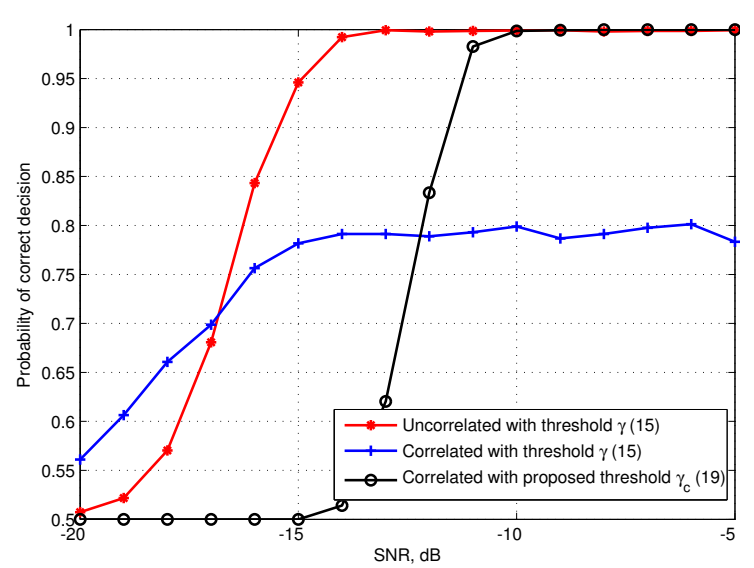

Fig. 3. Probability of correct decision versus $\operatorname{SNR}\left(N=100, \beta=2, P_{f}=\right.$ $0.07, \rho=0.5)$

variance expressions derived in Section 3.

While using the sensing threshold $\gamma$, given by (15), under the correlated noise case, the value of $P_{f}$ deviates from that of the target value used in determining the sensing threshold. This has been illustrated in Fig. 2, which shows the false alarm deviation versus correlation coefficient. From the figure, it can be noted that the proposed threshold $\gamma_{c}$, given by (19), compensates for this deviation and the difference in $P_{f}$ is almost zero as in the uncorrelated case while using the sensing threshold $\gamma$.

Figure 3 depicts the probability of correct decision versus SNR at a fixed false alarm rate of $P_{f}=0.07$ for 3 different cases with parameters $(N=100, \beta=2, \rho=0.5)$. From the figure, we note that although the correlated case with the threshold $\gamma$ performs better for low SNR values, it can not achieve the perfect sensing performance (even at higher SNR values). The perfect sensing is the condition in which there occur no false alarm and no miss detection. The correlated case with the proposed threshold $\lambda_{c}$ improves the sensing performance and achieves the perfect sensing performance above the SNR value of $-10 \mathrm{~dB}$. Therefore, it can be concluded that the proposed threshold for the ME based detection can be used for sensing under correlated noise scenarios.

\section{CONCLUSION}

In this paper, the maximum eigenvalue based detection was studied for sensing the PU signal under correlated noise scenarios. The theoretical analysis of the considered technique has been carried out under a noise only hypothesis using recent RMT results on the distribution of the maximum eigenvalue for a class of sample covariance matrices. Furthermore, an expression for the sensing threshold has been derived in terms of the probability of false alarm and system parameters. From the simulation results, it has been noted that the probability of false alarm for the considered correlated case increases with the increase in the correlation level and the proposed threshold compensates for this deviation. Hence, it can be concluded that the proposed sensing approach significantly improves the sensing performance in correlated noise scenarios.

\section{ACKNOWLEDGEMENT}

This work was supported by the National Research Fund, Luxembourg under AFR grant for a PhD project (Reference 3069102) and the CORE projects $\mathrm{CO}^{2} \mathrm{SAT}$ and SEMIGOD. 


\section{REFERENCES}

[1] A. Goldsmith, S. Jafar, I. Maric, and S. Srinivasa, "Breaking spectrum gridlock with cognitive radios: An information theoretic perspective,' proc. IEEE, vol. 97, no. 5, pp. 894 -914, May 2009.

[2] S. K. Sharma, S. Chatzinotas, and B. Ottersten, "Satellite cognitive communications: Interference modeling and techniques selection," in Proc. 6th ASMS/SPSC Conf., Sept. 2012, pp. $111-118$.

[3] E. Axell, G. Leus, E. G. Larsson, and H. V. Poor, "Spectrum Sensing for Cognitive Radio : State-of-the-Art and Recent Advances," IEEE Signal Processing Magazine, vol.29, no.3, pp.101 -116, May 2012.

[4] S. K. Sharma, S. Chatzinotas, and B. Ottersten, "Exploiting polarization for spectrum sensing in cognitive SatComs," in Proc. 7th Int. Conf. CROWNCOM, June 2012, pp. 36 -41.

[5] S. K. Sharma, S. Chatzinotas, and B. Ottersten, "Spectrum sensing in dual polarized fading channels for cognitive SatComs," in Proc. IEEE Globecom Conf., Dec. 2012, pp. 3419-3424.

[6] Y. Zeng and Y.-C. Liang, "Eigenvalue-based spectrum sensing algorithms for cognitive radio," IEEE Trans. Commun., vol. 57, no. 6, pp $1784-1793,2009$.

[7] W. Zhang, G. Abreu, M. Inamori, and Y. Sanada, "Spectrum sensing algorithms via finite random matrices," IEEE Trans. Commun., vol. 60, no. 1, pp. $164-175$, Jan. 2012.

[8] A. Kortun, T. Ratnarajah, M. Sellathurai, C. Zhong, and C. Papadias, "On the performance of eigenvalue-based cooperative spectrum sensing for cognitive radio," IEEE J. Selected Topics Signal Process., vol. 5, no. 1, pp. $49-55$, Feb. 2011

[9] J. Vinogradova, R. Couillet, and W. Hachem, "Statistical Inference in Large Antenna Arrays Under Unknown Noise Pattern," IEEE Trans. Signal Process., vol. 61, no. 22, pp.5633-5645, Nov. 2013.

[10] S. K. Sharma, S. Chatzinotas, and B. Ottersten, "Eigenvalue based sensing and SNR estimation for cognitive radio in presence of noise correlation," IEEE Trans. Veh. Technol., vol. 62, no. 8, pp. 3671 -3684, Oct. 2013 .

[11] L. Cardoso, M. Debbah, P. Bianchi, and J. Najim, "Cooperative spectrum sensing using random matrix theory," in proc. 3rd Int. Symp. Wireless Pervasive Comp., May 2008, pp. 334 -338.

[12] F. Penna, R. Garello, and M. Spirito, "Cooperative spectrum sensing based on the limiting eigenvalue ratio distribution in Wishart matrices," IEEE Commun. Letters, vol. 13, no. 7, pp. 507 -509, July 2009.

[13] S. K. Sharma, S. Chatzinotas, and B. Ottersten, "The effect of noise correlation on fractional sampling based spectrum sensing," in Proc IEEE ICC, June 2013, pp. $1182-1187$.

[14] Y. Zeng, C. Koh, and Y.-C. Liang, "Maximum eigenvalue detection: Theory and application," in Proc. IEEE ICC, May 2008, pp. $4160-$ 4164.

[15] P. Wang, J. Fang, N. Han, and H. Li, "Multiantenna-assisted spectrum sensing for cognitive radio," IEEE Trans. Veh. Technol., vol. 59, no. 4 pp. $1791-1800$, May 2010.

[16] P. Bianchi, M. Debbah, M. Maida, and J. Najim, "Performance of statistical tests for single-source detection using random matrix theory,' IEEE Trans. Info. Th., vol. 57, no. 4, pp. 2400 -2419, 2011.

[17] M. Matthaiou, M. Mckay, P. Smith, and J. Nossek, "On the condition number distribution of complex wishart matrices," IEEE Trans. Commun., vol. 58, no. 6, pp. 1705 -1717, June 2010.

[18] A. Kortun, M. Sellathurai, T. Ratnarajah, and C. Zhong, "Distribution of the ratio of the largest eigenvalue to the trace of complex wishar matrices," IEEE Trans. Signal Process., vol. 60, no. 10, pp. 5527 5532, Oct. 2012.

[19] S. Chatzinotas, S. K. Sharma, and B. Ottersten, "Asymptotic analysis of eigenvalue-based blind spectrum sensing techniques," in Proc. IEEE ICASSP, May 2013, pp. $4464-4468$.

[20] S. Chatzinotas, S. K. Sharma, and B. Ottersten, "Multiantenna signal processing for cognitive communications", in Proc. IEEE ChinaSIP, July 2013, pp. 293-297.
[21] S. K. Sharma, S. Chatzinotas, and B. Ottersten, "SNR estimation for multi-dimensional cognitive receiver under correlated channel/noise," IEEE Trans. Wireless Commun., vol. 12, no. 12, pp. 6392-6405, Dec. 2013.

[22] X. Mestre, J. Fonollosa, and A. Pages-Zamora, "Capacity of MIMO channels: asymptotic evaluation under correlated fading," IEEE J. Sel. Areas Commun., vol. 21, no. 5, pp. 829-838, 2003.

[23] S. K. Sharma, S. Chatzinotas, and B. Ottersten, "Eigenvalue based SNR Estimation for Cognitive Radio in Presence of Channel Correlation," in Proc. IEEE Globecom, Dec. 2013, pp. 1107-1112.

[24] S. Chatzinotas, M. Imran, and R. Hoshyar, "On the multicell processing capacity of the cellular MIMO uplink channel in correlated Rayleigh fading environment," IEEE Trans. Wireless Commun., vol. 8, no. 7, pp. $3704-3715$, July 2009.

[25] B. Nadler, F. Penna, and R. Garello, "Performance of Eigenvalue-Based Signal Detectors with Known and Unknown Noise Level," in Proc. IEEE Int. Conf. Commun., June 2011, pp. 1 -5.

[26] L. Wei, O. Tirkkonen, "Cooperative spectrum sensing of OFDM signals using largest eigenvalue distributions," in Proc. IEEE Personal, Indoor and Mobile Radio Commun., Sept. 2009, pp. 2295 -2299.

[27] Z. Bao, G. Pan, and W. Zhou "Universality for the largest eigenvalue of a class of sample covariance matrices," online, July 2013, http://arxiv.org/abs/1304.5690v4.

[28] N. El Karoui, "Tracy-Widom limit for the largest eigenvalue of a large class of complex sample covariance matrices", Ann. Probab. vol. 35, no. 2, pp. $663-714,2007$.

[29] I. M. Johnstone, "On the distribution of the largest eigenvalue in principal component analysis," Annals of Statistics, vol. 29, no. 2, pp. 295 $-327,2001$.

[30] C. Tracy and H. Widom, "On the distribution of the largest eigenvalue in principal component analysis," Comm. Math. Phys, vol. 177, pp. 727 $-754,1996$.

[31] A. M. Tulino and S. Verdu, "Random matrix theory and wireless communications," Foundations and Trends in Commun. and Inf. Th., vol. 1, no. 1, pp. 1-182, 2004.

[32] D. R. Joshi, D. C. Popescu, O. A. Dobre, "Adaptive spectrum sensing with noise variance estimation for dynamic cognitive radio systems," in Proc. 44th Annual Conf. Information Sciences and Systems, March 2010, pp. 1-5. 